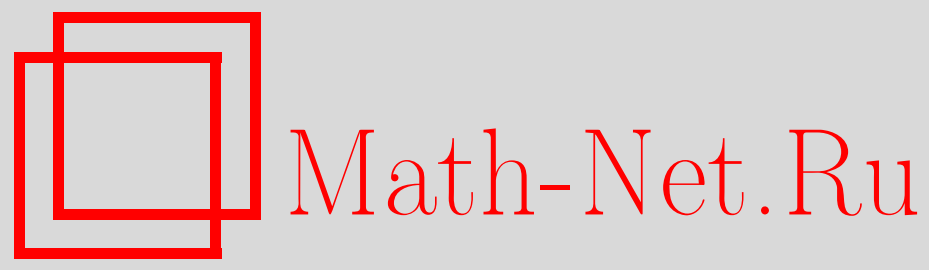

А. Н. Сергеев, Векторные и ковекторные инварианты супералгебр Ли, Функи. анализ и его прил., 1996, том 30, выпуск 3, 90-93

DOI: https://doi.org/10.4213/faa543

Использование Общероссийского математического портала MathNet.Ru подразумевает, что вы прочитали и согласны с пользовательским соглашением

http://www . mathnet.ru/rus/agreement

Параметры загрузки:

IP : 18.234 .197 .8

26 апреля 2023 г., 11:03:06

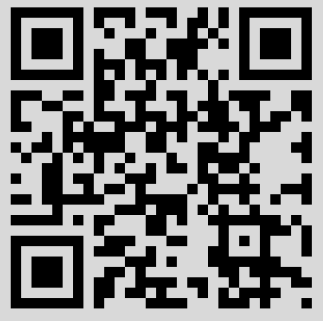


довательные его вершины $A, B, C$, не лежащие на одной прямой, расположен внутри угла $\angle A B C$.

СЛЕДСТВИЕ 4. Неособый регулярный многоугольник на плоскости с числом сторон, больиим трех, имеет не менее четьиех экстремальньх вершин.

Это утверждение является прямым обобщением теоремы [1] на случай невыпуклых многоугольников.

ОПРЕДЕЛЕНИЕ. Опорная вершина $V$ плоского многоугольника называется внутренней (внешней), если некоторые его вершины лежат внутри (вне) опорной окружности, проходящей через вершину $V$ и две ее соседние.

СЛЕДСТВИЕ 5 [6]. Выпукльй многоугольник на плоскости, не вписывающийся в окружность, имеет не менее двух внутренних и двух внешних вершин.

СЛЕДСТВИЕ 6. Любой несамопересекающийся регулярный многоугольник на плоскости либо вписьвается в окружность, либо имеет не менее двух внешних вершин.

Имеются и другие варианты теоремы о четырех вершинах для многоугольника (обзор см., например, в [7]). Автор благодарен О. Р. Мусину, заметившему, что условие регулярности плоского несамопересекающегося многоугольника эквивалентно тому, что этот многоугольник является подграфом триангуляиии Делоне множества своих вершин (см. [2, 5]).

\title{
ЛИтеРАТУРА
}

1. Мусин О. Р. Квант (1996) (в печати). 2. Препарата Ф., Шеймос М. Вычислительная геометрия: Введение. Мир, М., 1989. 3. Седых В. Д. Функц. анализ и его прил., 26, вып. 1, 35-41 (1992). 4. Седыл В. Д. Функц. анализ и его прил., 29, вып. 3, 41-50 (1995). 5. Guibas L., Stolfi J. ACM Trans. Graphics., 4, 74-123 (1985). 6. Schatteman A. Geom. Dedicata, 34, 229-242 (1990). 7. Wegner B. Math. Pannon., 6, No. 1, 121-132 (1995).

Московский государственный технологический университет «СТАНКИН»

Поступило в редакцию 25 декабря 1995 г.

\section{Векторные и ковекторные инварианты супералгебр Ли}

\author{
(C) 1996. A. H. СерГеЕB
}

Пусть $V$ - конечномерное суперпространство над $\mathbb{C}$ и $\mathfrak{g}$ - подсупералгебра Ли в $\mathfrak{g l}(V)$. В работе приводится описание образующих алгебр $\mathfrak{g}$-инвариантных элементов, содержащихся в $\mathfrak{A}_{k, l}^{p, q}=S\left(V^{k} \oplus \pi(V)^{l} \oplus V^{* p} \oplus \pi\left(V^{*}\right)^{q}\right)$. Полученные результаты являются обобщением работы [3], где аналогичное описание дано с точностью до операторов поляризации.

1. Инварианты супералгебры Ли $\mathrm{sl}(\boldsymbol{V})$. Для заданного суперпространства $E$ через $B(E)$ обозначим множество $\{1, \ldots, \alpha, \overline{1}, \ldots, \bar{\beta}\}$, где $\alpha=\operatorname{dim} E_{\overline{0}}$, 
a $\beta=\operatorname{dim} E_{\overline{1}}$, и выберем базис $\left\{e_{i}\right\}, i \in B(E)$, так, что четность базисного вектора совпадает с четностью соответствующего индекса. В пространстве $E^{*}$ выберем базис $\left\{e_{i}^{*}\right\}$ - левый дуальный к базису пространства $E$. Если $t$ - стандартная таблица Юнга (см. [2]), то через $e_{t}=\sum \varepsilon(\tau) \sigma \tau$ и $\tilde{e}_{t}=\sum \varepsilon(\tau) \tau \sigma$ обозначим минимальные идемпотенты в групповой алгебре симметрической группы, причем суммирование ведется по $\tau$ из столбцового стабилизатора и по $\sigma$ из строчного стабилизатора таблицы $t$. Для последовательности $I$ с элементами из $B(E)$ обозначим через $e_{I}$ элемент $e_{i_{1}} \otimes e_{i_{2}} \otimes \ldots$. Последовательность $I$ называется $t$-полустандартной, если при вписывании ее элементов в клетки таблицы $t\left(i_{\alpha}\right.$ вписывается в ту клетку, где стоит $\left.\alpha\right)$ ее элементы не убывают слева направо вдоль строк и сверху вниз по столбцам и, кроме того, четные элементы строго возрастают вдоль столбцов, а нечетные - вдоль строк. Обозначим через $\varphi_{E}$ канонический гомоморфизм тензорной алгебры $T(E)$ на симметрическую алгебру $S(E)$. Легко видеть, что $\mathfrak{A}_{k, l}^{p, q}=S\left(U \otimes V \oplus V^{*} \otimes W\right)$. Пусть $\left\{u_{\alpha}\right\},\left\{w_{\beta}\right\},\left\{v_{i}\right\},\left\{v_{i}^{*}\right\}$ - базисы в пространствах $U, W, V, V^{*}$ со- $^{-}$ ответственно. Для последовательностей $I$ и $J$ с элементами из $B(U)$ и $B(V)$ соответственно положим

$$
P_{t}^{U, V}(I, J)=\varphi_{U \otimes V}\left(e_{t}\left(u_{I}\right) \otimes v_{J}\right), \quad \widetilde{P}_{t}^{U, V}(I, J)=\varphi_{U \otimes V}\left(\tilde{e}_{t}\left(u_{I}\right) \otimes v_{J}\right) .
$$

Пусть $t$ - прямоугольная таблица размера $(n+k) \times m$, заполненная числами от 1 до $(n+k) m$ так, что вначале заполнена таблица, состоящая из первых $n$ строк, по столбцам, а затем оставшаяся часть также заполняется по столбцам. Пусть $s$ - прямоугольная таблица размера $n \times(m+k)$, заполненная последовательно по столбцам. Для последовательностей $I$ и $J$ через $I * J$ обозначим последовательность, полученную дописыванием к элементам последовательности $I$ элементов последовательности $J$. Через $I_{k}$ обозначим $k$-кратное повторение последовательности $12 \ldots n$, а через $J_{k}$ - последовательность, состоящую из $k$ символов $\overline{1}$, затем $k$ символов $\overline{2}$ и т.д. до $k$ символов $\bar{m}$, где $(n, m)=\operatorname{dim} V$. Обозначим через $d(I)$ число $(-1)^{p(I, I)}$, где $p(I, I)=\sum_{\beta>\alpha} p\left(i_{\alpha}\right) p\left(i_{\beta}\right)$, а $p$ функция четности, $p(I)=\sum p\left(i_{\alpha}\right)$.

TеоремА 1. Алгебра $\mathrm{sl}(V)$-инвариантных элементов порождена многочленами

(a) $\left(v_{\alpha}^{*}, v_{\beta}\right), \alpha \in B(U), \beta \in B(W)$ (cм. [3]);

(б) $F_{k}(S, T)=\sum d(L) P_{s}^{U, V}\left(S, I_{k} * L\right) \widetilde{P}_{t}^{V^{*}, W}\left(L * J_{k}, T\right)$;

(в) $F_{-k}(S, T)=\sum d(L)(-1)^{p(L) m k} \widetilde{P}_{t}^{U, V}\left(S, L * I_{k}\right) P_{s}^{V^{*}, W}\left(J_{k} * L, T\right)$,

где $k \in \mathbb{N}, S$ u $T$ суть $t$ - $u$ s-полустандартные последовательности $c$ элементами из $B(U)$ и $B(W)$ соответственно, а суммирование ведется по всем последовательностям $L$ длинь $\mathrm{nm}$ с элементами из $B(V)$.

2. Инварианты супералгебр Ли $\operatorname{osp}(\boldsymbol{V})$. Наличие четной инвариантной формы на модуле $V$ определяет изоморфизм $\mathfrak{A}_{k, l}^{p, q}=\mathfrak{A}^{p+k, q+l}$ как $\operatorname{osp}(V)$ модулей и как алгебр; поэтому можно ограничиться случаем $k=l=0$. Если $\operatorname{dim} V=(n, m)$, то для $i \in B(V)$ определим сопряженный $\tilde{i} \in B(V)$ по правилам: $\tilde{i}=n-i+1$, если $p(i)=\overline{0}$, и $\tilde{i}=\overline{m-i+1}$, если $p(i)=\overline{1}$. Пусть $t$ - прямоугольная таблица размера $n \times m$, заполненная по столбцам, и $L$ - последовательность с элементами из $t$. Заполним таблицу $t$ элементами последовательности $L\left(l_{\alpha}\right.$ стоит в той клетке, где было $\left.\alpha\right)$. Пару символов в $L$ 
назовем отмеченной, если они находятся в одной строке и в соседних столбцах, причем номер первого столбца нечетный. Обозначим через $\boldsymbol{T}_{1}$ множество последовательностей $L$, таких, что все отмеченные пары, кроме, возможно, пар последней строки, состоят из попарно сопряженных элементов; если из последней строки удалить все отмеченные пары с попарно сопряженными элементами, то оставшееся множество $N(L)=\left\{K_{1}, \ldots, K_{2 s}\right\}$ состоит из попарно различных нечетных элементов и инвариантно относительно сопряжения. Пусть $L \in \mathfrak{T}_{1}$; тогда определены числа $d(L)=d\left(L_{1} * L_{2}\right) d\left(L_{3} * L_{4}\right) \ldots$, где $L_{1}, L_{2}, \ldots$, последовательные столбцы последовательности $L$ и

$$
K(L)=\sum_{q=s}^{s+\nu}(k+1)^{r} 2^{r-q}(r-q) ! k^{q} \sigma_{q-s}\left(k_{1}, \ldots, k_{\nu}\right),
$$

где $r=m / 2, \nu$ - количество различных отмеченных пар последней строки, состоящих из попарно сопряженных нечетных элементов, не принадлежащих множеству $N(L), k_{1}, \ldots, k_{\nu}$ - кратности, с которыми эти пары входят в последнюю строку, $k=k_{1}+\cdots+k_{\nu}$ и $\sigma_{l}$ - элементарная симметрическая функция.

Tеорема 2. Алгебра оsp $(V)$-инвариантных многочленов порождена элементами

(a) $\left(v_{\alpha}, v_{\beta}\right), \alpha, \beta \in B(W)($ c.⿲. [3]);

(б) $R(J)=\sum d(L) K(L) P_{s}^{V^{*}, W}\left(I_{1} * L, J\right)$,

причем суммирование ведется по $L \in \mathfrak{T}_{1}, s$ - прямоугольная таблица размера $n \times(m+1)$, заполненная по столбцам, $u$ J есть s-полустандартная последовательность с элементами из $B(W)$.

3. Инварианты супералгебры Ли $\operatorname{sp}(V)$. Наличие нечетной инвариантной билинейной формы на пространстве $V$ определяет изоморфизм $\mathfrak{A}_{k, l}^{p, q}=$ $\mathfrak{A}^{p+l, q+k}$ как $\operatorname{sp}(V)$-модулей и как алгебр; поэтому можно ограничиться случаем $k=l=0$. Так как $\operatorname{dim} V_{\overline{0}}=\operatorname{dim} V_{\overline{1}}$, то для $i \in B(V)$ определен сопряженный элемент $\bar{i}$. Обозначим через $\boldsymbol{T}_{2}$ множество последовательностей длины $n^{2}$, pacсматриваемых как $n \times n$-таблицы, заполненные по столбцам, которые обладают следуюшими свойствами: по главной диагонали стоят числа $\overline{1}, \ldots, \bar{n}$; выше главной диагонали стоят четные числа и числа, симметричные относительно главной диагонали, сопряжены; на пересечении $i$-й строки и $j$-го столбца стоит одно из чисел $\{i, j, \bar{i}, \bar{j}\}$.

Для $I \in \mathfrak{T}_{2}$ пусть $\varepsilon(I)=(-1)^{\nu}$, где $\nu$ - количество четных чисел в $I$, не совпадающих с номером своего столбца, $m_{k}(I)=\prod_{1}^{n} \frac{(n+k) !}{\left(n+k-k_{i}\right) !}$, где $k \geqslant 0$ и $k_{i}$ - количество чисел в $i$-м столбце, не равных $i$ и $\bar{i}$. Пусть $t$ и $s$ - такие же таблицы, как в теореме 1 .

ТЕОРемА 3. Алгебра $\operatorname{sp}(V)$-инвариантных элементов порождена многочленами

(i) $\left(v_{\alpha}, v_{\beta}\right), \alpha, \beta \in B(W)($ cм. [3]);

(ii) $Q_{k}(J)=\sum \varepsilon(I) m_{k-1}(I) P_{t}^{V^{*}, W}\left(I * J_{k-1}, J\right)$;

(iii) $Q_{-k}(J)=\sum \varepsilon(I) m_{0}(I) P_{s}^{V^{*}, W}\left(I * I_{k+1}, J\right)$,

где $k \geqslant 1$ и J есть $t$ - $(s-)$ стандартнал последовательность с элементами из $B(W)$ и суммирование ведется по $I \in \mathfrak{T}_{2}$. 


\section{ЛИТЕРАТУРА}

1. Вейль Г. Классические группы, их инварианты и представления. ИЛ, М. (1947). 2. Макдональд И. Симметрические функции и многочлены Холла. Мир, М. (1985). 3. Сергеев А. Н. Функц. анализ и его прил., 26, вып. 3, 88-90 (1992).

Балаковский институт техники, технологии и управления

Поступило в редакцию 15 декабря 1994 г.

УДК 517.98

\section{Об одной теореме о неподвижной точке}

(c) 1996. ЧАн Куок Бинь, Нгуен Минь ЧыОнг

1. ТеОРема 1. Пусть $H$ - замкнутое ограниченное множество в нормированном пространстве $X$ и $T$ - отображение множества $\mathrm{H} \times \mathrm{H}$ в $\mathrm{H}$, удовлетворяющее следующему условию:

$$
\|T(x, y)-T(z, t)\| \begin{cases}<\max \{\|x-z\|,\|y-t\|\}, & \text { если }(x, y) \neq(z, t) \\ \leqslant\|x-z\|=\|y-t\|, & \text { и } x \neq y \text { или } z \neq t, \\ \leqslant \text { сли } x=z u z=t,\end{cases}
$$

при всех $x, y, z, t \in H($ cм. $[5,6])$.

Предполагается, кроме того, что либо $H$ компактно, либо $T$ - отображение множества $H \times H$ в компактное подмножество множества $H$.

Тогда уравнение

$$
T(x, x)=x
$$

имеет единственное решение в Н. Кроме того, каждое уравнение

$$
x_{n}=T\left(x_{n}, x_{n-1}\right), \quad n \geqslant 1,
$$

имеет единственное решение $x_{n}$ и последовательность $\left\{x_{n}\right\}$, определенная уравнением (3), сходитсл к решению уравнения (2) при любом выборе точки $x_{0} \in H$.

Эта теорема доказывается без труда при помощи теоремы Эдельстейна из [3] для отображения $T_{v}(x)=T(x, v)$.

Заметим, что настоящая теорема распространяет теорему Эдельстейна из [3] на отображение $T(\cdot, \cdot)$. Если вместо $(3)$ в тех же предположениях, что и в теореме 1 , использовать итерацию Пикарда для $T_{*}(x)=T(x, x)$, как это сделано в работах $[1-3,10]$ и др., то нельзя будет говорить о существовании и сходимости приближенных решений для уравнения (2).

2. Аналогично доказательству теоремы 1 доказывается

Теорема 2. Пусть $H$ - замкнутое ограниченное подмножество в метрическом пространстве $(X, d)$ u $g(\cdot, \cdot): H \times H \rightarrow[0, \infty)-\oint у н к ц и я$, обладаюшая следуюшими свойствами:
а) $g(x, y)=0$, если и только если $x=y$ для всех $x, y \in H$;
б) $g$ непрерьвна по $(x, y)$;
в) если $g(x, y) \rightarrow 0$, mo $d(x, y) \rightarrow 0$. 